\title{
PROTON AND DEUTERON RELAXATION STUDY OF MOLECULAR DYNAMICS IN LYSOZYME SOLUTIONS
}

\author{
J.F. KAKUlE ${ }^{a}$, W.P. WęGLARz ${ }^{b}$, R.K. ShenOY ${ }^{c *}$, A.R. ShaRP ${ }^{a}$ \\ AND H. PEEMOELleR ${ }^{c}$ \\ ${ }^{a}$ Department of Physics, University of New Brunswick, Fredericton \\ New Brunswick, E3B 5A3, Canada \\ ${ }^{b} \mathrm{H}$. Niewodniczański Institute of Nuclear Physics, Kraków, Poland \\ ${ }^{c}$ Department of Physics, University of Waterloo, Waterloo, Ontario, N2L 3G1, Canada
}

(Received August 23, 1999; revised version May 15, 2000)

\begin{abstract}
A nuclear magnetic resonance spin-lattice relaxation dispersion study of the relaxation of several magnetization components in both natural and deuterated lysozyme solutions was undertaken at $20^{\circ} \mathrm{C}$. Proton and deuteron resonances were employed. The two-dimensional time evolution of the magnetization and the spin-spin relaxation were analyzed. In addition, an isotopic dilution study was performed at 5 and $30.6 \mathrm{MHz}$. The results indicate that the water proton spin-lattice relaxation rate which arises from intermolecular relaxation between the water protons and the lysozyme protons represents a relatively strong relaxation mechanism. A model for the dynamics of the water molecules, consistent with the proton and deuteron dispersions as well as with the isotopic dilution results, is presented.
\end{abstract}

PACS numbers: 87.15.-v, 76.60.-k,

\section{Introduction}

It has been recognized [1] that the interaction of protein molecules and solvent molecules in aqueous solutions influences the dynamics of both as well as the conformation of the proteins. There is a considerable interest in understanding details of these interactions on the molecular level, as they are the source of tissue contrast in magnetic resonance imaging [2].

Nuclear magnetic resonance (NMR) provides a probe of both the local magnetic environment and motions of spin bearing molecules. NMR has therefore been applied extensively to the study of molecular dynamics and intermolecular interactions in protein solutions [3-29]. In particular, NMR relaxation studies of the solvent have given information about the solute-solvent interactions. However,

\footnotetext{
*Permanent address is Fonar Corporation, 110 Marcus Drive, Melville, New York, 11747,
} U.S.A. 
there is still a considerable interest in understanding the relaxation mechanisms responsible for the observed water nuclei relaxation rates in aqueous protein solutions.

The observed proton spin-lattice relaxation rate $\left(R_{1}=1 / T_{1}\right)$ of protein solutions, is expected to be a composite of the protein proton rate $\left(R_{\mathrm{p}}\right)$ and the water proton rate $\left(R_{\mathrm{w}}\right)$. Since the protein molecules are heterogeneous $R_{\mathrm{p}}$ itself is a sum of contributions from various spin groups located in different structural and dynamic environments. A common protein relaxation rate $R_{1}$ is observed as a result of fast "magnetization transfer" among spin groups [10].

The water is also dynamically heterogeneous consisting of at least two phases. Water molecules whose dynamics is modified by being associated with protein molecules are often denoted as being in a "bound" phase. The remaining water molecules are assumed to have dynamics similar to molecules in the bulk liquid. To take account of this, a fast-exchange-two-state (FETS) model $[3,4,9]$ is commonly assumed.

Spin communication may also occur across the protein-water interface in the form of proton exchange between proteins and water, or of magnetic coupling (cross-relaxation) between the protein protons and the bound water protons. This spin communication, if fast, means that protons on both water and proteins can share their individual relaxations to the lattice. Thus, the intermolecular water-protein relaxation mechanism contributes directly to the observed spin-lattie relaxation of both water and protein protons.

It is fundamental to the understanding of the molecular dynamics in these systems to separate the various contributions to the observed relaxation rates. In particular, it is crucial to determine the role played by the protein-water intermolecular spin-spin communication. For example, if this communication is negligible, the water protons are relaxed by the water dynamics only and information about their dynamics could be obtained directly from a relaxation study of the water protons.

This problem has been extensively addressed in the literature [2-26]. However, different conclusions have been reached.

Intermolecular communication across the protein-water interface was found to be very important in one study of lysozyme solutions [6], important in other studies [18, 21], and negligible in yet another study of lysozyme solutions [9].

In this paper an attempt to resolve this controversy by elucidating in detail the different mechanisms responsible for the relaxation of water protons in hen-egg-white lysozyme (HEWL) solutions is presented. To this end, proton $T_{1}$ in HEWL solutions as a function of the degree of solvent deuteration, both proton and deuteron $T_{1}$ as a function of Larmor frequency, and spin-spin relaxation times $\left(T_{2}\right)$ in a few selected cases are reported.

\section{Experimental methods}

\subsection{Samples}

Grade $1 \mathrm{HEWL}$, disodium ethylenediamine tetraacetate (EDTA), and $99.8 \%$ $\mathrm{D}_{2} \mathrm{O}$ were all purchased from the Sigma Chemical Company. Salts and paramagnetic impurities were removed from the HEWL by dialyzing at $14^{\circ} \mathrm{C}$ against 
$0.01 \mathrm{M}$ EDTA in distilled $\mathrm{H}_{2} \mathrm{O}$ (see Refs. $[8,19]$ for details). The resulting HEWL-water solution was lyophilized and the dry powder was stored at $-5^{\circ} \mathrm{C}$.

To prepare deuterated HEWL, $0.5 \mathrm{~g}$ of this powder was dissolved in $50 \mathrm{ml}$ of $99.8 \% \mathrm{D}_{2} \mathrm{O}$ and 8 hours were allowed for labile protons to exchange before the sample was lyophilized. The deuteration process was then repeated a second time using the powder from the first deuteration. The final deuterated powder was stored in an air-tight vessel at $-5^{\circ} \mathrm{C}$.

All glassware used, including the thin walled $8 \mathrm{~mm}$ outside diameter NMR sample tubes, was cleaned to remove metal impurities [8, 19]. Paramagnetic oxygen was removed from the water used in the preparation of the natural HEWL solution (N-HEWL) by a freeze-pump-thaw technique. Within the experimental accuracy, the $T_{1}$ of the purified water sample after exposure to the air for about one minute (the time required to transfer the water from its container to the HEWL sample) was the same as the published $T_{1}$ for pure water at the same temperature.

The actual samples were then prepared in the following manner. About $50 \mathrm{mg}$ of HEWL was placed in a previously weighed sample tube. The sample was then dried under a vacuum of about $10^{-3}$ Torr for 10 hours and reweighed to determine the weight of the dry sample. The appropriate quantity of the purified water was then added and the sample was flame sealed. Ten other samples were also prepared in which the water was isotopically diluted with $\mathrm{D}_{2} \mathrm{O}$ to various extents. The deuterated HEWL (D-HEWL) solutions were prepared in the same way except that the deuterated HEWL powder and $\mathrm{D}_{2} \mathrm{O}$ was used. The $\mathrm{pH}$ of the solutions was $6.6 \pm 0.1$.

The N-HEWL samples were $10.4 \mathrm{mM}$ or $87 \% \mathrm{H}_{2} \mathrm{O}$ and $13 \%$ natural HEWL by weight. The D-HEWL solutions were also $10.4 \mathrm{mM}$ or $87.6 \% \mathrm{D}_{2} \mathrm{O}$ and $12.4 \%$ deuterated HEWL. The samples used in the isotopic dilution experiment were $(10.4 \pm 0.2) \mathrm{mM}$, with a proton mole fraction $X_{\mathrm{H}}$ ranging from 0.1 to 1 . In calculating $X_{\mathrm{H}}$ labile $\mathrm{HEWL}$ protons were taken into account.

The commercially obtained heavy water, labelled $99.8 \% \mathrm{D}_{2} \mathrm{O}$, was found to be $(99.7 \pm 0.1 \%) \mathrm{D}_{2} \mathrm{O}$. This implies that the $\mathrm{D}-\mathrm{HEWL}$ solution does not contain more than $0.2 \%$ of $\mathrm{H}_{2} \mathrm{O}$ by weight.

\subsection{Measurements}

All measurements were performed using a home built broad band pulsed NMR spectrometer capable of operating over the $5-100 \mathrm{MHz}$ frequency range. The frequency source for the spectrometer was a PTS 250 synthesizer (Programmed Test Sources, Inc.). The deuteron relaxation experiments below $15 \mathrm{MHz}(2.3 \mathrm{~T})$ and all proton relaxation experiments utilized a Varian HA100 magnet. For the deuteron relaxation experiments above $15 \mathrm{MHz}$ a Nalorac 200 superconducting magnet was used. The data were digitized with a Datalab 912 transient wave form recorder.

The inversion recovery sequence $\left(180^{\circ}-\tau-90^{\circ}\right)$ was used for the $T_{1}$ measurements. $T_{2}$ was measured with the Carr-Purcell-Meiboom-Gill (CPMG) sequence with a pulse spacing of $1 \mathrm{~ms}$. These pulse sequences were used for both proton and deuteron relaxation experiments. 
In selected cases, the evolution of the magnetization was monitored along two time axes [30]. This form of two-dimensional time evolution (2DTE) correlation spectroscopy, is called two-dimensional time domain (2DTD) NMR [30, 31]. It involves the reconstruction of the free induction decays (FIDs) of the various magnetization components of the system from correlated spin-lattice relaxation measurements.

All measurements were performed at $293 \mathrm{~K}$.

\section{Results}

The proton CPMG spin-echo envelopes of the D-HEWL and N-HEWL samples are shown in Fig. 1a and b, respectively. In the N-HEWL solution the decay was decomposed into two magnetization components, one liquid-like $\left(L_{1}\right)$ with
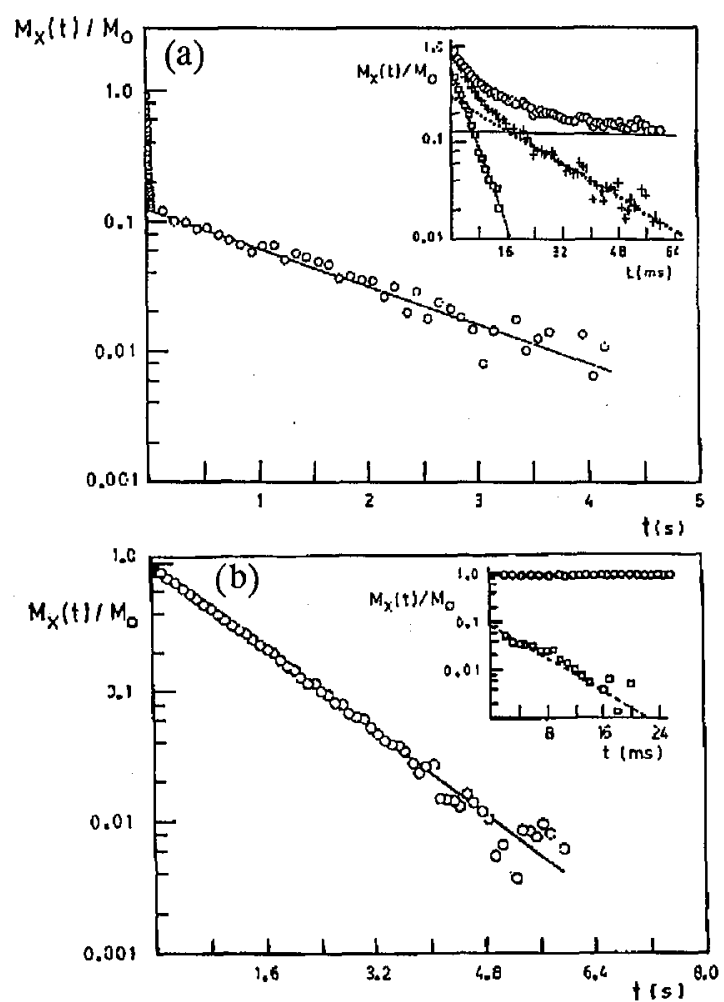

Fig. 1. The main part of the proton spin-echo envelopes of HEWL solutions. (a) The experimental decay curve of D-HEWL fitted to $M_{x}(t) / M_{0}=0.13 \exp (-t / 1500)+$ $0.30 \exp (-t / 20)+0.57 \exp (-t / 4.2)$. The inset shows the decomposition of the composite decay curve into components $L_{1}$ (solid line), $L_{2}$ (dotted line), and $S L$ (dashed line). (b) The experimental decay curve of N-HEWL fitted to $M_{x}(t) / M_{0}=$ $0.93 \exp (-t / 1100)+0.07 \exp (-t / 5.1)$. The inset shows the initial $30 \mathrm{~ms}$ of the decay. The time $t$ is in milliseconds. 
TABLE I

The proton magnetization relaxation times in HEWL solutions at $40 \mathrm{MHz}$ and at room temperature.

\begin{tabular}{c|c|c|c|c|c}
\hline \hline Exp. & Spin group & Magnetization & Relaxation & \multicolumn{2}{|c}{ 2D time evolution } \\
\cline { 4 - 6 } & character & fraction [\%] & time & $\begin{array}{c}\text { Magnetization } \\
\text { fraction [\%] }\end{array}$ & $T_{2}[\mathrm{~ms}]$ \\
\hline \multicolumn{5}{c}{ N-HEWL solution } \\
\hline$T_{2}$ & $L_{1}$ & $93 \pm 1$ & $1.1 \pm 0.1 \mathrm{~s}$ & & \\
& $L_{2}$ & & & \\
& $S L$ & $7 \pm 1$ & $5.1 \pm 0.5 \mathrm{~ms}$ & & \\
\hline$T_{1}$ & $L_{1}$ & & $1.8 \pm 0.1 \mathrm{~s}$ & $92 \pm 2$ & $\sim 15^{*}$ \\
& $L_{2}$ & & & & \\
& $S L$ & & $80 \pm 10 \mathrm{~ms}$ & $8 \pm 2$ & $5.1 \pm 0.5$ \\
\hline
\end{tabular}

D-HEWL solution

\begin{tabular}{c|c|c|c|c|c}
\hline$T_{2}$ & $L_{1}$ & $13 \pm 1$ & $1.5 \pm 0.1 \mathrm{~s}$ & & \\
& $L_{2}$ & $30 \pm 1$ & $20 \pm 1 \mathrm{~ms}$ & & \\
& $S L$ & $57 \pm 1$ & $4.2 \pm 0.2 \mathrm{~ms}$ & & \\
\hline$T_{1}$ & $L_{1}$ & & $4 \pm 1 \mathrm{~s}$ & $8 \pm 1$ & $\sim 15^{*}$ \\
& $L_{2}$ & & \} $110 \pm 10 \mathrm{~ms}$ & $92 \pm 1$ & $4.2 \pm 0.2$
\end{tabular}

$T_{2}=1100 \mathrm{~ms}$ and one semiliquid-like $(S L)$ with $T_{2}=5.1 \mathrm{~ms}$ (Table I). In the D-HEWL, two similar magnetization components were observed along with a third component which was liquid-like $\left(L_{2}\right)$ having $T_{2}=20 \mathrm{~ms}$ (Table I). The size fractions of the various components are also given in Table $\mathrm{I}$.

In both D- and N-HEWL the proton magnetization recovery curves at the shorter time windows showed two-component behaviour. The values of $T_{1}$ are plotted as a function of the FID window for D-HEWL in Fig. 2a and for N-HEWL in Fig. 3a. The magnetization component FIDs reconstructed from the 2DTD are given in Figs. $2 \mathrm{~b}$ and $3 \mathrm{~b}$, respectively. In each case, the $T_{1}$ values of a particular magnetization component were averaged over all time windows. The average values are given in Table I, in the column "2D time evolution".

From the 2DTD experiment, it is apparent that the spin group with the larger value of $T_{1}$ is also characterized by the larger value of $T_{2}$ in each sample. An exponential was fitted to each corresponding decay curve (solid lines in Figs. $2 \mathrm{~b}$ and $3 \mathrm{~b}$ ) and in each case $T_{2}$ was about $15 \mathrm{~ms}$. This value is considerably smaller than the $T_{2}$ of the liquid-like components found in the CPMG experiments (Table I). This is caused by the inhomogeneity of the main field because $T_{2}$ was also about $15 \mathrm{~ms}$ for a pure water sample. We therefore label these long $T_{2}$ 's observed in the 2DTE experiment as $15^{*} \mathrm{~ms}$ (see Table I). Such $T_{2}$ 's are usually denoted as $T_{2}^{*}$.

In D-HEWL, the reconstructed FID curve for the spin group with the short $T_{1}$ (Fig. 2b) exhibits nonexponential behaviour and decays more rapidly than the 

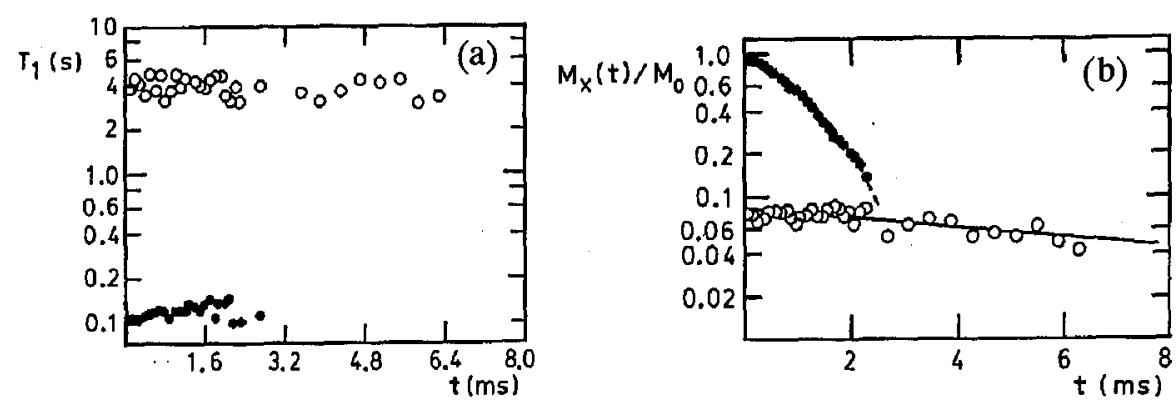

Fig. 2. 2DTE experiment in D-HEWL at $40 \mathrm{MHz}$. (a) $T_{1}$ plotted as a function of window position. The long and short $T_{1}$ 's are represented by the open and filled circles, respectively. (b) Reconstructed FIDs of spin groups with long $T_{1}$ (open circles) and short $T_{1}$ (filled circles). The dashed line was calculated from $M_{x}(t) / M_{0}=0.92 \exp [-(t / 4.2)-$ $\left.0.28 t^{2}\right]$, where $t$ is in ms. The sizes of the magnetization fractions and the decay time constants are given in Table I.
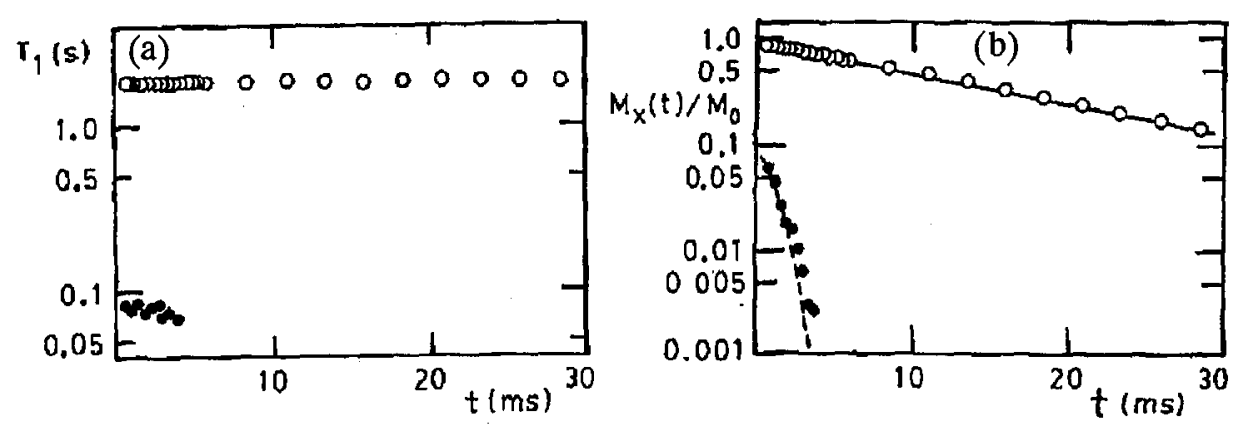

Fig. 3. 2DTE experiment in N-HEWL at $40 \mathrm{MHz}$. (a) $T_{1}$ plotted as a function of window position. The long and short $T_{1}$ 's are represented by the open and filled circles, respectively. (b) Reconstructed FIDs of spin groups with long $T_{1}$ (open circles) and short $T_{1}$ (filled circles). The dashed line was calculated from $M_{x}(t) / M_{0}=0.08 \exp [-(t / 5.2)-$ $0.28 t^{2}$ ], where $t$ is in ms. Table I gives the sizes of the magnetization fractions and decay time constants.

CPMG spin-echo envelope for the same spin group (Fig. Ia and Table I). This is because many proton groups with different chemical shifts reside on a HEWL molecule causing a dephasing of the transverse magnetizations of these groups. For simplicity, a Gaussian distribution of chemical shifts was assumed and the above decay curve was approximated by $M_{x}(t)=M_{0} \exp \left(-t / T_{2}-c t^{2}\right)$, where $c$ is a constant which describes the width of the distribution of the chemical shifts. The dashed line in Fig. 2b was calculated from this expression using a least-squares curve fitting routine [32] with $c$ and $M_{0}$ as the adjustable parameters and the value of $T_{2}$ for the $S L$-component (Table I). The "best fit" parameters are given in the caption of Fig. $2 \mathrm{~b}$.

In N-HEWL, the reconstructed decay curve (Fig. 3b) was treated similarly using the corresponding $T_{2}$ of the $S L$-component, the constant $c$ from the above calculation, and $M_{0}$ as the adjustable parameter. The dashed line in Fig. 3b rep- 
resents the best fit, with the parameters given in the figure caption. These results from the 2DTD experiment are summarized in Table I, column " $2 \mathrm{D}$ time evolution".

In addition to the above 2DTD experiments, proton $T_{1}$ dispersion was measured in both $\mathrm{N}$ - and $\mathrm{D}$-HEWL at about 10 frequencies in the range of $5-100 \mathrm{MHz}$ at the 100 microsecond window on the FID. Two-component behaviour was observed in the recovery curves at each frequency. The size of the magnetization components obtained from the decompositions was independent of frequency and similar to the size of the corresponding fraction determined in the 2DTE experiment at $40 \mathrm{MHz}$ (Table I).

Figure 4 shows $1 / T_{1}$ of the fast relaxing component in D-HEWL plotted as a function of frequency $(\nu)$. The values of $1 / T_{1}$ for the $L_{1}$-component exhibited considerable scatter but no frequency dependence was observed. The dispersion of the proton $1 / T_{1}$ of the $S L$ - and $L_{1}$-components in N-HEWL is shown in Figs. 5 and $6 \mathrm{a}$, respectively. Figure $6 \mathrm{~b}$ shows the values of $1 / T_{1}$ of deuterons in D-HEWL in the frequency range of $4-30.6 \mathrm{MHz}$. In this case, at each frequency, the recovery curves were fitted to a single exponential.

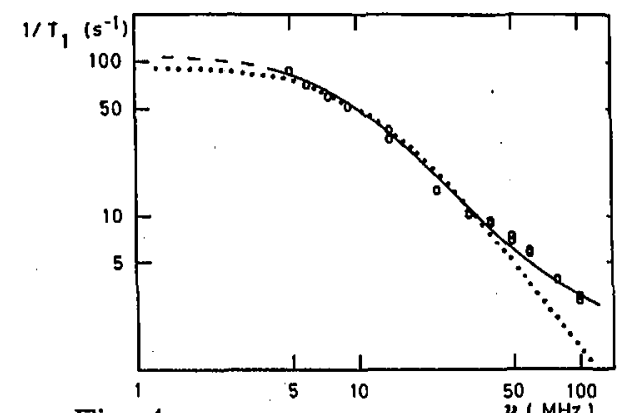

Fig. 4

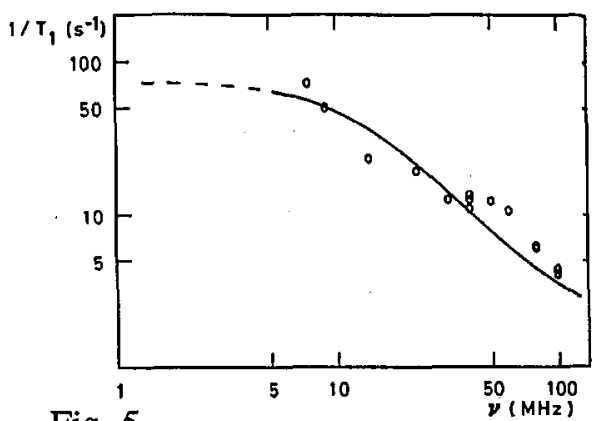

Fig. 5

Fig. 4. Proton $T_{1}$ dispersion of the $S L$-, $L_{2}$-components in D-HEWL. The dotted and solid lines were calculated from Eqs. (2) and (4), respectively. See the text and Table III for details.

Fig. 5. Proton $T_{1}$ dispersion of the $S L$-component in N-HEWL. The solid line represents the best fit of Eq. (4) to the data.

In part, the proton $1 / T_{1}$ dispersion of the $L_{1}$-component in N-HEWL and the deuteron $1 / T_{1}$ dispersion of the $L_{1}$-component in D-HEWL have been reported in the literature $[21,33]$. The measurements in the literature extend to lower frequencies, whereas our measurements include data at higher frequencies. There is good agreement with the published work where the results overlap.

The observed proton rates $R_{1 \mathrm{OBS}}\left(X_{\mathrm{H}}\right)$ of the $L_{1}$-component in HEWL solutions are plotted in Fig. 7 as a function of the proton mole fraction $\left(X_{\mathbf{H}}\right)$ of the solvent at resonance frequencies of $5 \mathrm{MHz}$ and $30.6 \mathrm{MHz}$. At each frequency, $T_{1}$ of the fast relaxing component was independent of $X_{\mathbf{H}}$. These values were averaged to obtain $T_{1}(5 \mathrm{MHz})=(10 \pm 2) \mathrm{ms}$ and $T_{1}(30.6 \mathrm{MHz})=(70 \pm 3) \mathrm{ms}$. These $T_{1}$ 's correspond to the $T_{1}$ 's of the $S L$-component at the appropriate frequency as found 

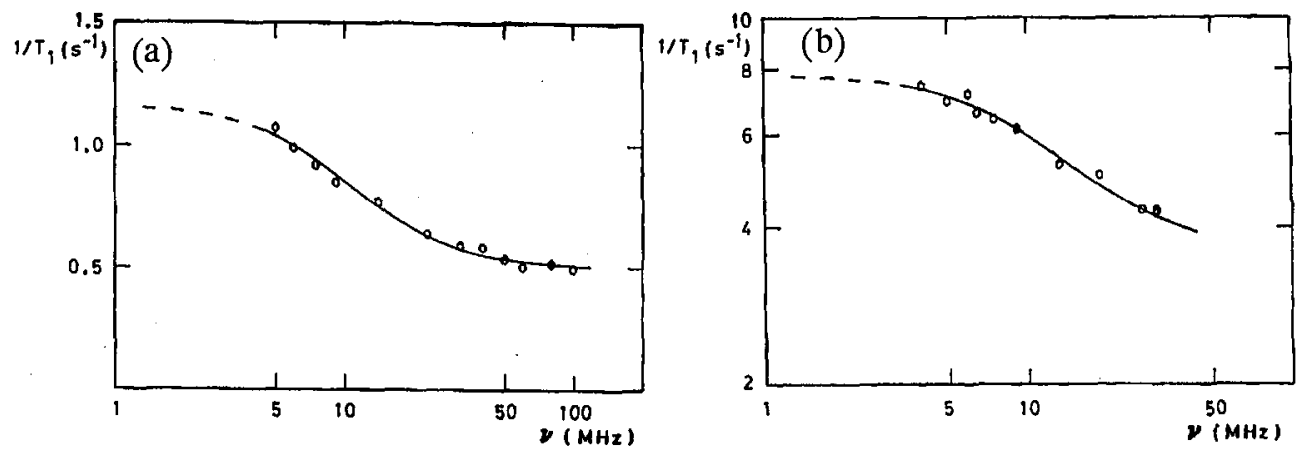

Fig. 6. $T_{1}$ dispersion of the $L_{1}$-component; (a) proton $T_{1}$ in N-HEWL, (b) deuteron $T_{1}$ in D-HEWL. In each case the solid line represents the best fit using Eq. (5).
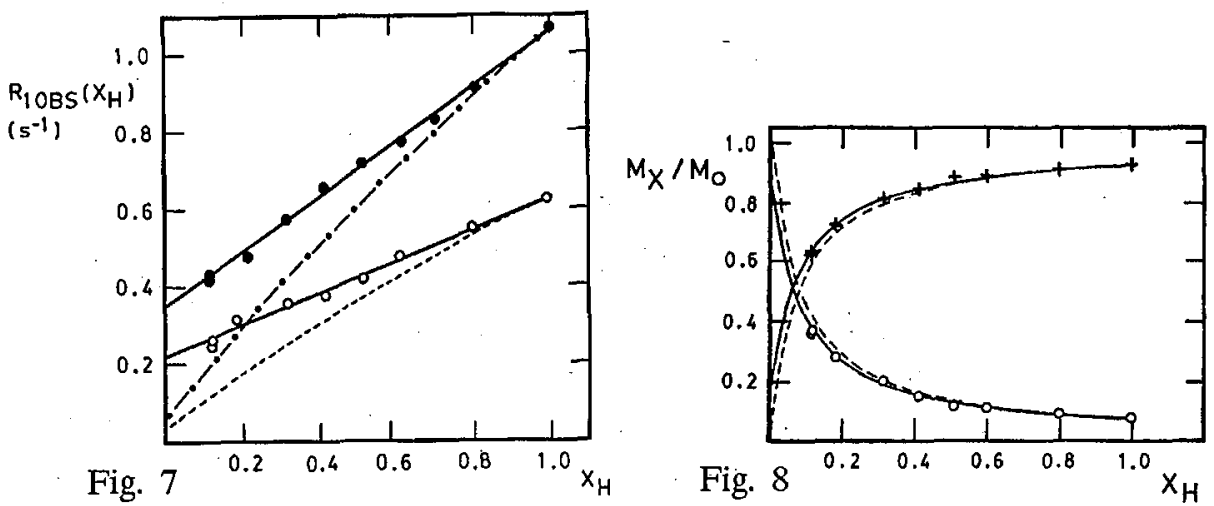

Fig. 7. $R_{1 \text { OBS }}$ of the $L_{1}$-component plotted as a function of $X_{\mathrm{H}}$ at $5 \mathrm{MHz}$ (filled circles) and $30.6 \mathrm{MHz}$ (open circles). The dot-dashed line and dashed line represent the corresponding relaxation rates expected in the absence of a HEWL proton - water proton intermolecular contribution. The solid lines were calculated from Eq. (1) with $A=0.70, B=0.35$ for $\nu=5 \mathrm{MHz}$, and $A=0.41, B=0.22$ for $\nu=30.6 \mathrm{MHz}$.

Fig. 8. Fractions of the $L_{1}$-component (crosses) and the fast relaxing component (open circles) versus $X_{\mathrm{H}}$ at $30.6 \mathrm{MHz}$. The solid lines are calculated from Eqs. (7) and the dashed lines from Eqs. (7), without the contribution due to the $L_{1}$-component observed in D-HEWL.

in the dispersion experiment on N-HEWL (Fig. 5). The normalized fractions of the $L_{1}$-component and of the fast relaxing component are shown for the isotopically diluted solutions in Fig. 8 for $30.6 \mathrm{MHz}$.

In the absence of a HEWL proton-water proton intermolecular contribution the observed water proton rate $R_{1 \mathrm{OBS}}\left(X_{\mathrm{H}}\right)$ would be expected to vary with $X_{\mathrm{H}}$ in a manner similar to that in the bulk mixture as shown in Fig. 7 (dot-dashed and dashed lines). The experimental values of $R_{1 \mathrm{OBS}}\left(X_{\mathrm{H}}\right)$ (Fig. 7), however, decrease more slowly with decreasing $X_{\mathrm{H}}$ than these predicted values. This indicates that a rate, which is independent of the dilution, contributes to $R_{1 \mathrm{OBS}}\left(X_{\mathrm{H}}\right)$ at each frequency. 
TABLE II

Relaxation rates in $\mathrm{N}-\mathrm{HEWL}$, at $293 \mathrm{~K}$, obtained from the isotopic dilution experiment.

\begin{tabular}{l|c|c}
\hline \multicolumn{1}{c|}{ Frequency } & $5 \mathrm{MHz}$ & $30.6 \mathrm{MHz}$ \\
\hline$R_{10 \mathrm{BS}}\left(X_{\mathrm{H}}=1\right)$ & $1.04 \pm 0.07$ & $0.63 \pm 0.02$ \\
$P_{\mathrm{wp}} R_{1 \mathrm{w}-\mathrm{p}}$ & $0.32 \pm 0.02$ & $0.20 \pm 0.01$ \\
$R_{1 \mathrm{w}}$ & $0.73 \pm 0.04$ & $0.43 \pm 0.03$ \\
$\left(1-f_{\mathrm{wb}}\right) R_{1 \mathrm{wf}}$ & 0.31 & 0.31 \\
$f_{\mathrm{wb}} R_{1 \mathrm{wb}}$ & $0.41 \pm 0.04$ & $0.12 \pm 0.03$ \\
\hline
\end{tabular}

Note: All relaxation rates are in $s^{-1}$. Values of $\left(1-f_{\mathrm{wb}}\right) R_{1 \mathrm{wf}}$ and $f_{\mathrm{wb}} R_{1 \mathrm{wb}}$ have been estimated as discussed in the text.

In general the variation of $R_{1 \mathrm{OBS}}\left(X_{\mathrm{H}}\right)$ with $X_{\mathrm{H}}$ is expected to be nonlinear because the solvent viscosity varies with $X_{\mathrm{H}}$, yet the data (Fig. 7 ) vary linearly with $X_{\mathrm{H}}$. With the assumption that the viscosity corrections can be neglected the observed rate is given by

$$
R_{10 \mathrm{BS}}\left(X_{\mathrm{H}}\right)=A X_{\mathrm{H}}+B
$$

where $A=23 R_{1 \mathrm{w}} / 24, B=R_{1 \mathrm{w}} / 24+P_{\mathrm{wp}} R_{1 \mathrm{w}-\mathrm{p}}, R_{1 \mathrm{w}}$ is the water proton spin-lattice relaxation rate, $R_{1 \mathrm{w}-\mathrm{p}}$ is the contribution to the rate of a water proton due to its interaction with nonexchangeable protein protons and $P_{\mathrm{wp}}$ is the fraction of water protons taking part in such interaction.

The solid lines in Fig. 7 were calculated from Eq. (1) using the linear least-squares method with the values of $A$ and $B$ given in the figure caption (Fig. 7). $R_{1 \mathrm{w}}$ and $P_{\mathrm{wp}} R_{1 \mathrm{w}-\mathrm{p}}$ calculated from Eq. (1) using these values of $A$ and $B$ are listed in Table II. Comparison of estimates of $P_{\mathrm{wp}} R_{1 \mathrm{w}-\mathrm{p}}$ to the corresponding $R_{1 \mathrm{OBS}}\left(X_{\mathrm{H}}=1\right)$ (see Fig. 7 and Table II) shows that a protein proton water proton intermolecular relaxation mechanism contributes about $30 \%$ to the observed rate in $\mathrm{N}$-HEWL at each frequency.

\section{Discussion}

The proton spin density of $\mathrm{H}_{2} \mathrm{O}$ is 1.66 times that of HEWL. (Calculated using water and the known HEWL structure [34].) This means that N-HEWL sample stoichiometry predicts that the water protons and the protein protons contribute $91.7 \%$ and $8.3 \%$ to the sample magnetization, respectively. Within experimental error the magnetization fractions of the $L_{1^{-}}$and $S L$-components obtained from the $T_{2}$ experiment and 2DTD experiment in N-HEWL (Table I) are the same as predicted from stoichiometry. This, taken together with the fact that the $T_{2}$ 's of the $S L$-components in N- and D-HEWL are essentially the same (Table I), leads us to conclude that in N-HEWL the $L_{1}$ - and $S L$-components can be associated with the water and HEWL protons, respectively.

These results indicate no mixing of HEWL proton magnetization and water proton magnetization (separate FIDs from 2DTD experiments). Thus, neither 
spin-spin coupling (or cross-relaxation) across the interface nor exchange of labile protein hydrogens with water hydrogens appear to occur to any appreciable extent, and in N-HEWL one is justified in associating the $S L$-component with the HEWL protons and the $L_{1}$-component with the water protons.

In the 2DTE experiment the sizes of the FIDs reconstructed from magnetization components recovering with the long and short $T_{1}$ (see Fig. 2b) correspond well to sizes obtained in the $T_{2}$ experiment in D-HEWL for the $L_{1}$-component and the sum of the $S L$ - and $L_{2}$ - components, respectively (Table I). Furthermore, the shapes of the reconstructed FIDs (Fig. $2 \mathrm{~b}$ ) correspond well with the $T_{2}$ 's found in the $T_{2}$ experiment when the line broadening due to field inhomogeneity is taken into account. These observations in D-HEWL lead us to associate the FIDs reconstructed from the long $T_{1}$ with the $L_{1}$-component and those reconstructed from the short $T_{1}$-components with the $S L$ - and $L_{2}$-components.

The $L_{2}$-component was observed in D-HEWL only (Table I). It likely comes about as follows. Upon deuteration a number of labile protons on the protein are replaced by deuterons. Any protein proton groups which had been able to communicate with the rest of the protein protons through these labile protons become more isolated in D-HEWL. Thus, in D-HEWL, some protons relax at their own intrinsic rate as they do not communicate with the other protein protons, giving the $L_{2}$-component observed in D-HEWL, but not in N-HEWL.

It is rather interesting that in D-HEWL a liquid-like proton component is observed with a $T_{2}$ that is greater than that of the $L_{1}$-component (water) in N-HEWL (Table I). This component might be associated with either (a) water protons present in the deuterated solvent as an impurity or (b) protons on parts of the HEWL molecule which are very mobile in solution as well as isolated from other HEWL protons.

The possibility (a) was eliminated as follows. After the completion of the NMR experiments in D-HEWL, the solvent was evaporated in a vacuum system and collected in a cold trap. This distillate was checked for proton content using NMR. It was found that any residual proton content in the solvent would account for no more than about $1 \%$ of the total proton magnetization observed in D-HEWL. This is less than one tenth of the size fraction of the $L_{1}$-component observed in D-HEWL (Table I). We conclude that the case (b) is applicable.

It is not clear at this time if the same $L_{1}$-magnetization component seen in D-HEWL is also present in N-HEWL because it would be impossible to resolve it in N-HEWL for two reasons. It is only of the order of $1 \%$ as compared to $93 \%$ for the $L_{1}$-magnetization component in N-HEWL, and its $T_{1}$ is only about twice that of the water protons in N-HEWL.

\section{1. $T_{1}$ dispersion}

For an isotropically reorienting proton pair separated by a constant distance $r$ the spin-lattice relaxation rate $R_{1}\left({ }^{1} \mathrm{H}\right)$ is given by [35]

$$
R_{1}\left({ }^{1} \mathrm{H}\right)=\frac{3 \gamma^{4} \hbar^{2}}{10 r^{6}} F\left(\tau_{c}\right)
$$


where

$$
F\left(\tau_{\mathrm{c}}\right)=\frac{\tau_{\mathrm{c}}}{1+\omega^{2} \tau_{\mathrm{c}}^{2}}+\frac{4 \tau_{\mathrm{c}}}{1+4 \omega^{2} \tau_{\mathrm{c}}^{2}}
$$

$\gamma$ is the proton gyromagnetic ratio, $\hbar$ - Planck's constant divided by $2 \pi, \omega-$ the Larmor frequency and $\tau_{c}$ - the correlation time characterizing the motion. The model for the dynamics of the interproton vector upon which $\mathrm{Eq}$. (2) is based is clearly too simple to accurately account for the HEWL proton dynamics and interactions. Nevertheless, it is instructive to apply it to the HEWL $S L-, L_{2}$-component data obtained in D-HEWL (Fig. 4) to estimate the HEWL tumbling time. Providing $r$ (Eq. (2)) is treated as an effective interproton distance and there is no major intra-HEWL molecular motion, this should yield a reasonable estimate. The dotted line (Fig. 4) was calculated from Eq. (2) using a least-squares curve fitting routine [32] with $r$ and $\tau_{c}$ as adjustable parameters. The "best fit" parameters are $r=1.4 \AA$ and $\tau_{\mathrm{c}}=20 \mathrm{~ns}$. Clearly the fit is not very good, particularly in the high frequency region of the dispersion curve. This discrepancy likely is the result of the omission of a contribution from intramolecular protein dynamics with high frequency components (relative to the experimental frequency range).

Segments of the HEWL molecule have been shown to undergo displacements on less than nanosecond time scales [36]. In addition, methyl groups on protein molecules in solution undergo relatively rapid rotation about their threefold axis of symmetry [10]. The effect of the latter on HEWL relaxation is relatively easily taken into account and we consider it first.

Let the rapid methyl rotation be characterized by a correlation time $\tau_{\mathrm{CH}_{3}}$. The threefold axis will itself reorient with characteristic time $\tau_{\mathrm{c}}$ as the protein molecule tumbles in solution. Assuming fast magnetization transfer between the $\mathrm{CH}_{3}$ groups and the other protein protons, the HEWL proton rate $R_{1 \mathrm{p}}$ becomes

$$
R_{1 \mathrm{p}}=f_{\mathrm{CH}_{3}} R_{1 \mathrm{CH}_{3}}+\left(1-f_{\mathrm{CH}_{3}}\right) R_{1} \text { (other } \mathrm{H}^{\prime} \mathrm{s} \text { ). }
$$

In this expression $f_{\mathrm{CH}_{3}}$ is the HEWL proton fraction due to $\mathrm{CH}_{3}$ groups; $R_{1 \mathrm{CH}_{3}}$ is given [9] by

$$
R_{1 \mathrm{CH}_{3}}=\frac{0.6 \gamma^{4} \hbar^{2}}{r_{\mathrm{CH}}^{6}}\left[0.25 F\left(\tau_{\mathrm{c}}\right)+0.75 F\left(\tau_{c_{3}}\right)\right]
$$

with $\tau_{\mathrm{c3}}^{-1}=\tau_{\mathrm{c}}^{-1}+\tau_{\mathrm{CH}_{3}}^{-1}$ and $r_{\mathrm{CH}_{3}}$ is the interproton distance within the $\mathrm{CH}_{3}$ group; $R_{1}$ (other H's) is the spin-lattice relaxation rate of other HEWL protons involved in the relaxation and is given by Eq. (2). Equation (4) was fitted to the data (Fig. 4) using $f_{\mathrm{CH}_{3}}=0.19$ and $r_{\mathrm{CH}_{3}}=1.78 \AA$ (known from the HEWL structure [34]) with $r$ and $\tau_{\mathrm{c}}$ as adjustable parameters. The fit was repeated for several values of $\tau_{\mathrm{CH}_{3}}$ using $\tau_{\mathrm{CH}_{3}}=0.1 \mathrm{~ns}[10]$ as a starting point. The "best fit" (solid line, Fig. 4) was obtained for $\tau_{\mathrm{CH}_{3}}=(0.30 \pm 0.05) \mathrm{ns}$. The values of the effective HEWL interproton distance and $\tau_{\mathrm{c}}$ used in the calculation are given in Table III.

In view of the fact that the fit obtained using Eq. (4), with fixed, stoichiometric $f_{\mathrm{CH}_{3}}$ provides a reasonable fit to the data (solid line, Fig. 4) suggests that high frequency HEWL segment displacements contribute to HEWL proton $T_{1}$ to a smaller extent. This is likely the consequence of many such displacements having only small amplitudes. For purposes of the following we neglect this contribution 
TABLE III

$T_{1}$ Dispersion results in N- and D-HEWL solutions at $293 \mathrm{~K}$.

\begin{tabular}{|c|c|c|c|c|}
\hline Nucleus & $\begin{array}{l}\text { Spin group } \\
\text { character }\end{array}$ & $\begin{array}{c}\tau_{\mathrm{c}} \\
{[\mathrm{ns}]}\end{array}$ & $f_{\mathrm{wb}}$ & $\begin{array}{c}\text { effective } \\
r[\AA]\end{array}$ \\
\hline \multicolumn{5}{|c|}{ N-HEWL } \\
\hline \multirow[t]{3}{*}{${ }^{1} \mathrm{H}$} & $L_{1}$ & $9 \pm 2$ & $(1.4 \pm 0.1) \times 10^{-3}$ & \\
\hline & $L_{2}$ & & & \\
\hline & $S L$ & $7 \pm 1$ & & $2.1 \pm 0.1$ \\
\hline \multicolumn{5}{|c|}{ D-HEWL } \\
\hline \multirow[t]{2}{*}{${ }^{1} \mathrm{H}$} & $L_{1}$ & & & \\
\hline & $\begin{array}{l}L_{2} \\
S L\end{array}$ & \} $10 \pm 1$ & & \} $2.1 \pm 0.1$ \\
\hline${ }^{2} \mathrm{H}$ & $\overline{L_{1}}$ & $\overline{8 \pm 2}$ & $(0.68 \pm 0.08) \times 10^{-3}$ & \\
\hline
\end{tabular}

and consider Eq. (4), with $\tau_{\mathrm{c}}$ and $\tau_{\mathrm{CH}_{3}}$, to be a reasonable approximation for HEWL relaxation.

From the $T_{2}$ and 2DTE results it was deduced that the $S L$-spin group in N-HEWL consists of HEWL protons. Therefore, the $T_{1}$ dispersion data of the $S L$-component in N-HEWL (Fig. 5) was analysed in a manner similar to the analysis of the $S L$ - and $L_{2}$-component data in D-HEWL (solid line, Fig. 4). The solid line (Fig. 5) was calculated from Eq. (4) using the same values of $f_{\mathrm{CH}_{3}}$ and $\tau_{\mathrm{CH}_{3}}$ with $r$ and $\tau_{\mathrm{c}}$ as adjustable parameters (see Table III for final values). The fit (Fig. 5) is not as good as that in Fig. 4 (solid line) due to the larger scatter of points defining the $S L$-component in N-HEWL compared to that in D-HEWL. (The fractional size of the $S L$-component in N-HEWL is only $\sim 0.07$ whereas in D-HEWL the $S L$ - and $L_{2}$-components amount to $\sim 0.92$ of the total magnetization.)

The value of $\tau_{\mathrm{c}}$ of the $S L$-spin group in N-HEWL is somewhat smaller than that in D-HEWL (Table III). Recalling that the viscosity of $\mathrm{D}_{2} \mathrm{O}$ is $25 \%$ larger than that of $\mathrm{H}_{2} \mathrm{O}$ [37], the two values of $\tau_{\mathrm{c}}$ may be considered to be in agreement.

The reasonable agreement between the results obtained from the $S L$-component data in N-HEWL and those obtained from the $S L$ - and $L_{2}$-component data in D-HEWL (see Table III) supports the proposal made earlier that the $S L$-spin group in N-HEWL consists of HEWL protons.

The relaxation rates of water nuclei in protein solutions are often analyzed using the fast-exchange-two state (FETS) model. According to this model the observed water nuclei spin-lattice relaxation rate $R_{1 \mathrm{w}}$ is the weighted sum of the rate of water molecules "bound" to the protein $\left(R_{1 \mathrm{wb}}\right)$ and the rate of the remaining bulk-liquid-like water in the sample $\left(R_{1 \mathrm{wf}}\right)$ as follows

$$
R_{1 \mathrm{w}}=f_{\mathrm{wb}} R_{1 \mathrm{wb}}+\left(1-f_{\mathrm{wb}}\right) R_{1 \mathrm{wf}},
$$

where $f_{\mathrm{wb}}$ is the fraction of "bound" water. Equation (5) was fitted to the data (Fig. 6a) using a least-squares curve fitting routine [32] with $R_{1 \mathrm{wb}}\left({ }^{1} \mathrm{H}\right)$ taken from 
Eq. (2) and adjustable parameters $f_{\mathrm{wb}}, \tau_{\mathrm{c}}$ (see Table III), and $R_{1 \mathrm{wf}}\left({ }^{1} \mathrm{H}\right)$. The water molecule interproton distance $r=1.58 \AA$. The fit is good (solid line, Fig. 6a).

The correlation time of the bound water molecules $\left(\tau_{\mathrm{c}}=9 \mathrm{~ns}\right.$ from Table III) corresponds well with that of the $S L$-spin group in N-HEWL (Table III) indicating that the water molecules acquire the HEWL molecule dynamics. In addition, $R_{1 \text { wf }}\left({ }^{1} \mathrm{H}\right)=(0.5 \pm 0.1) \mathrm{s}^{-1}$ is greater than $R_{1}$ bulk water which equals $0.32 \mathrm{~s}^{-1}$ at $293 \mathrm{~K}$ [38]. This suggests that some of the water molecules in the solution are dynamically influenced by the protein molecules such that their motion is slower than in the bulk liquid, however, still fast enough that their proton $T_{1}$ does not depend on frequency in the $5-100 \mathrm{MHz}$ region [39].

Unfortunately, the observed water proton rates cannot be used directly to deduce dynamical information about the water molecules in protein solutions with absolute confidence due to possible contributions from water proton - protein proton intermolecular coupling that may produce spin-lattice relaxation and/or magnetization exchange effects [40-42]. In addition, exchange of labile protein hydrogens may play an important role [29]. However, it has been shown [33] that in lysozyme solution, at $\mathrm{pH} \sim 7$, labile protein hydrogens do not make any measurable contribution to solvent proton relaxation rates, and this effect is assumed negligible in the present study.

An attractive alternative for studying water dynamics in protein solutions is to use $\mathrm{D}_{2} \mathrm{O}$ in place of $\mathrm{H}_{2} \mathrm{O}$ and observe deuteron resonance. Since the deuteron relaxes primarily by means of intramolecular quadrupolar interactions, the intermolecular spin-spin coupling which complicates the interpretation of the proton relaxation experiments is negligible [43]. The deuteron $T_{1}$ dispersion data (Fig. 6b) was analysed with the FETS model (Eq. (5)), where $R_{1 \mathrm{wb}}\left({ }^{2} \mathrm{H}\right)$ is given [35] by

$$
R_{1}\left({ }^{2} \mathrm{H}\right)=\frac{3 \pi^{2}}{10}(Q C C)^{2} F\left(\tau_{\mathrm{c}}\right)
$$

with $Q C C$ being the quadrupolar coupling constant. In Fig. $6 \mathrm{~b}$ the solid line represents the best fit of Eq. (5) to the data with adjustable parameters $f_{\mathrm{wb}}, \tau_{\mathrm{c}}$ (see Table III), and $R_{1 \mathrm{wf}}\left({ }^{2} \mathrm{H}\right)$. In the calculation the bulk water $Q C C=230 \mathrm{kHz}$ [44] was used.

The value of $\tau_{\mathrm{c}}=(8 \pm 2) \mathrm{ns}$, obtained from the $T_{1}\left({ }^{2} \mathrm{H}\right)$ experiment (Fig. $6 \mathrm{~b}$ ), is in agreement with the value of $\tau_{\mathrm{c}}$ for HEWL molecule tumbling (Fig. 4 and Table III). This suggests that some water molecules must be bound to the HEWL molecule for a time at least of the order $\tau_{c}$ because in the $T_{1}\left({ }^{2} \mathrm{H}\right)$ experiment (Fig. 6b) the water cannot acquire information about the protein dynamics by means of HEWL-water spin communication.

The apparent free water rate $R_{1 \text { wf }}\left({ }^{2} \mathrm{H}\right)=(3.6 \pm 0.3) \mathrm{s}^{-1}$ found from the fit (Fig. 6b) is approximately $1 \mathrm{~s}^{-1}$ larger than the rate $R_{1}=2.6 \mathrm{~s}^{-1}$ for bulk $\mathrm{D}_{2} \mathrm{O}$ at $293 \mathrm{~K}$ [45]. This result is consistent with the suggestion made in connection with $R_{1 \text { wf }}\left({ }^{1} \mathrm{H}\right)$ in N-HEWL that certain water molecules move more slowly than in the bulk liquid, but still fast enough to show no dispersion at frequencies below $100 \mathrm{MHz}$.

The value of $f_{\mathrm{wb}}$ in N-HEWL is about twice as large as $f_{\mathrm{wb}}$ in D-HEWL (Table III). The hydrogen bond energy involving the deuteron is slightly larger than that involving the proton, and replacing $\mathrm{H}_{2} \mathrm{O}$ by $\mathrm{D}_{2} \mathrm{O}$ is not expected to 
make substantial changes to the bonding details (e.g. the number of bound water molecules, residence times, etc.) We propose that the above difference in $f_{\mathrm{wb}}$ results from a relaxation mechanism that contributes to the water proton rate but not to the water deuteron rate, and was not taken explicitly into account when the FETS model (Eq. (5)) was applied (Fig. 6a and b). The calculated bound water fraction in N-HEWL therefore appears larger than it in fact is.

\subsection{Isotopic dilution}

It has been indicated [12] that a water proton - protein proton intermolecular mechanism should not be neglected when information about molecular dynamics is deduced from relaxation data in protein solutions. Koenig et al. [24-26] have shown that an intermolecular (protein-water) cross-relaxation mechanism contributes significantly to the water proton relaxation rate in several protein solutions, including some in which the solvent was diluted with $\mathrm{D}_{2} \mathrm{O}$. A similar conclusion was reached by Blicharska et al. [6] for lysozyme and egg white solutions. In addition, the same authors showed [6] that the smaller values of $T_{1}$ (relative to $T_{1}$ in pure water) observed in protein solutions cannot be due to the paramagnetic ions present in such solutions.

In a proton NMR isotopic dilution study [18] in lysozyme solutions it was concluded that the bound water protons as well as the nonexchangeable protein protons contribute to the observed water relaxation rate in a major way. A similar conclusion was reached in [21], where direct dipole-dipole interaction was suggested as the dominating mechanism.

In principle, the isotopic dilution approach is ideally suited for determining the water-HEWL intermolecular contribution. This is because the number of water protons in a sample can be progressively decreased in a known fashion to determine the presence or absence of the above contribution at variable water proton concentrations.

Comparison of values of $P_{\mathrm{wp}} R_{1 \mathrm{w}-\mathrm{p}}$, obtained from the fit of Eq. (1) to the relaxation rate dilution data (Fig. 7$)$ to $R_{1 \mathrm{OBS}}\left(X_{\mathrm{H}}=1\right)$ values indicated a HEWL-water intermolecular relaxation contribution of $30 \%$ to $R_{1 \text { OBS }}$ in N-HEWL (see Sec. 3 and Table II). An additional proof that this intermolecular contribution $\left(P_{\mathrm{wp}} R_{1 \mathrm{w}-\mathrm{p}}\right)$ to $R_{1 \mathrm{OBS}}$ does not come from the protein magnetization through the spin-spin coupling of the two magnetizations can be realized from a consideration of the relative magnetization magnitudes of the fast relaxing component and the $L_{1}$-component in the isotopic dilution experiments (Fig. 8). Assuming that the water proton - protein proton cross-relaxation effects are negligible, the normalized water proton magnetization fraction $M_{\mathrm{w}}\left(X_{\mathrm{H}}\right)$ and protein proton magnetization fraction $M_{\mathrm{p}}\left(X_{\mathrm{H}}\right)$ are given by

$$
M_{\mathrm{w}}\left(X_{\mathrm{H}}\right)=\frac{X_{\mathrm{H}} M_{\mathrm{w}}(1)+0.13\left(1-X_{\mathrm{H}}\right) M_{\mathrm{p}}(1)}{M_{\mathrm{p}}(1)+X_{\mathrm{H}} M_{\mathrm{w}}(1)}
$$

and

$$
M_{\mathrm{p}}\left(X_{\mathrm{H}}\right)=\frac{M_{\mathrm{p}}(1)-0.13\left(1-X_{\mathrm{H}}\right) M_{\mathrm{p}}(1)}{M_{\mathrm{p}}(1)+X_{\mathrm{H}} M_{\mathrm{W}}(1)}
$$


where $M_{\mathrm{w}}(1)$ and $M_{\mathrm{p}}(1)$ are the respective stoichiometric water and protein equilibrium magnetizations at $X_{\mathrm{H}}=1$, and 0.13 is the fraction of the $L_{1}$-component in D-HEWL (see Table I). The solid lines in Fig. 8 were calculated from Eqs. (7) using $M_{\mathrm{w}}(1)=0.917$ and $M_{\mathrm{p}}(1)=0.083$. There is close agreement between the experimental magnetization fractions and those calculated, without adjustable parameters. (The dashed lines were calculated from Eqs. (7) without the $L_{1}$-component observed in D-HEWL taken into account.) Except for somewhat more scatter in the data, the relative magnetization magnitudes of the two components at $5 \mathrm{MHz}$ varied with $X_{\mathrm{H}}$ very much like those at $30.6 \mathrm{MHz}$ (Fig. 8). The above represents an additional proof that the spin-spin coupling of the HEWL and water magnetizations cannot be very strong.

\subsection{Model for water molecule dynamics in HEWL solutions}

In order to gain insight into the water molecule dynamics at the HEWL-water interface, we investigate the frequency dependence of (a) the water proton contribution and (b) the contribution of the water proton-HEWL proton intermolecular coupling to the observed rate $R_{1 \mathrm{OBS}}\left(X_{\mathrm{H}}=1\right)$ of the $L_{1}$-component in N-HEWL. The weighted water proton rates $\left(1-f_{\mathrm{wb}}\right) R_{1 \mathrm{wf}}$ as well as $R_{10 \mathrm{BS}}\left(X_{\mathrm{H}}=1\right)$ and $R_{1 \mathrm{~W}}$ at $5 \mathrm{MHz}$ and $30.6 \mathrm{MHz}$ (from Table II) are shown in Fig. 9. Also shown are the values of the proton and deuteron spin-lattice relaxation rates (taken from Fig. 6) of the $L_{1}$-component in N-HEWL and D-HEWL, respectively.

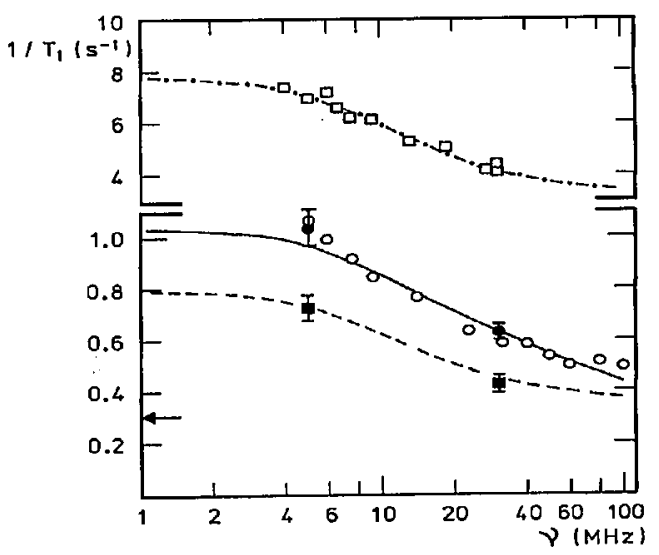

Fig. 9. Dispersion of experimental rates $R_{1}$ of the $L_{1}$-component in $\mathrm{N}$-HEWL (open circles - for protons taken from Fig. 6a) and in D-HEWL (open squares - for deuterons taken from Fig. 6b), and of calculated values of $R_{1 \mathrm{w}} . R_{1 \mathrm{w}}$ of protons (dashed line) and of deuterons (dashed-dotted line) were calculated from Eq. (5) with $R_{1 \mathrm{wb}}$ of Eq. (8) and fitted parameters $f_{\mathrm{wb}}=(3.8 \pm 0.6) \times 10^{-3}, \tau_{f}=(0.23 \pm 0.06)$ ns and $f_{1}=0.70 \pm 0.05$. The solid line represents values of $\lambda^{-}$calculated from Eq. (10), assuming that all of the water molecules in the first hydration layer communicate across the water-HEWL interface. Also shown are the weighted rate $\left(1-f_{\mathrm{wb}}\right) R_{1 \mathrm{wf}}$ (horizontal arrow), as well as $R_{1 \mathrm{OBS}}\left(X_{\mathrm{H}}=1\right)$ (filled circles) and $R_{1 \mathrm{w}}$ (filled squares) at 30.6 and $5 \mathrm{MHz}$. 
If a water-HEWL relaxation mechanism is involved, detailed information about the water proton - HEWL proton interfacial interactions would be required to accurately predict the dependence of $R_{1 \mathrm{w}-\mathrm{p}}$ on frequency. Although such information is not available, it is possible to estimate the relative importance of such a contribution to $R_{1 \mathrm{w}-\mathrm{p}}$. We proceed by first estimating the frequency dependence of the intrinsic water proton rate $R_{1 \mathrm{w}}\left({ }^{1} \mathrm{H}\right)$ by utilizing parameters for the model of water molecule dynamics derived from the deuteron data $\left(R_{1 \mathrm{w}}\left({ }^{2} \mathrm{H}\right)\right.$, Figs. $6 \mathrm{~b}$ and 9 ) and requiring that the estimated values of $R_{1 \mathrm{w}}\left({ }^{1} \mathrm{H}\right)$ are consistent with the results obtained from the isotopic dilution experiments at $5 \mathrm{MHz}$ and $30.6 \mathrm{MHz}$ (Fig. 9).

In an early NMR relaxation dispersion study [11] of bovine serum albumin solutions it was concluded that an isotropic motion model for the dynamics of bound water molecules was not consistent with all the experimental results. In the following NMR studies $[46,47]$ of hydrated HEWL powder it was shown that the water of hydration undergoes anisotropic motion. For purposes of the present discussion we assume that a small fraction $f_{\mathrm{wb}}$ (not necessarily the same as the one considered above) of water molecules undergoes anisotropic motion while attached to the HEWL molecule via hydrogen bonds for a time at least as long as the protein tumbling time. The remaining bound water molecules making up a "general hydration sphere" are assumed to be restricted in their translational diffusive motion as a result of the presence of the protein surface. The rotational diffusive motion of these water molecules is assumed to be sufficiently fast so as to contribute a non-dispersive component to the relaxation rate. This assumption is reasonable as can be seen from the following.

A monolayer coverage of the HEWL molecule involves approximately 160 water molecules (a hydration level of $0.23 \mathrm{~g} \mathrm{H}_{2} \mathrm{O} / \mathrm{g}\left(\mathrm{HEWL}+\mathrm{H}_{2} \mathrm{O}\right.$ )) [33]. The fraction of bound water needed to explain the intrinsic water rate $R_{1 \mathrm{w}}\left({ }^{2} \mathrm{H}\right)$ was found to be $(0.68 \pm 0.08) \times 10^{-3}$ (Table III), which amounts to about 3 water molecules per HEWL molecule. If we assume that the water molecules in question undergo anisotropic motion at a binding site (rather than being irrotationally bound), this fraction is expected to increase. However, it is not expected to increase by orders of magnitude. This suggests that the rotational dynamics of most of the water molecules in the first hydration layer must not be strongly linked to the protein dynamics.

Bound water molecules are expected to form hydrogen bonds with either oxygens or nitrogens on the HEWL as depicted in Fig. 10. The fractions of bound water molecules in position (1) and in position (2) are denoted as $f_{1}$ and $\left(1-f_{1}\right)$, respectively. The motion of these water molecules is characterized by $\tau_{f}$, the time scale for rotation of the water molecule about the hydrogen bond direction (also referred to as "fast rotation axis"), and by $\tau_{s}$, the rate of reorientation of the fast rotation axis. Then $R_{1 \mathrm{wb}}$ is given [48] by

$$
\begin{aligned}
R_{1 \mathrm{wb}} & =\left[A_{1} f\left(\tau_{s}\right)+B_{1} f\left(\tau_{1}\right)+C_{1} f\left(\tau_{2}\right)\right] f_{1} \\
+ & {\left[A_{2} f\left(\tau_{s}\right)+B_{2} f\left(\tau_{1}\right)+C_{2} f\left(\tau_{2}\right)\right]\left(1-f_{1}\right) / 2 } \\
+ & {\left[A_{3} f\left(\tau_{s}\right)+B_{3} f\left(\tau_{1}\right)+C_{3} f\left(\tau_{2}\right)\right]\left(1-f_{1}\right) / 2, }
\end{aligned}
$$


where, for $i=1,2,3$,

$$
\begin{aligned}
& A_{i}=(1 / 4)\left(3 \cos ^{2} \theta_{i}-1\right)^{2}, \quad B_{i}=3 \sin ^{2} \theta_{i} \cos ^{2} \theta_{i}, \quad C_{i}=(3 / 4) \sin ^{4} \theta_{i}, \\
& f(\tau)=Q\left[\tau /\left(1+\omega^{2} \tau^{2}\right)+4 \tau /\left(1+4 \omega^{2} \tau^{2}\right)\right], \\
& Q=3 \gamma^{4} \hbar^{2} / 10 r^{6} \quad \text { for protons and } 3 \pi^{2}(Q C C)^{2} / 10 \text { for deuterons, } \\
& \tau_{1}^{-1}=\tau_{s}^{-1}+\tau_{f}^{-1}, \quad \tau_{2}^{-1}=\tau_{s}^{-1}+4 \tau_{f}^{-1},
\end{aligned}
$$

and $\theta$ is the angle between the fast rotation axis and the water molecule internuclear vector for $\mathrm{N}-\mathrm{HEWL}$, and between the fast rotation axis and the direction of the electric field gradient at the site of the water deuteron for D-HEWL.

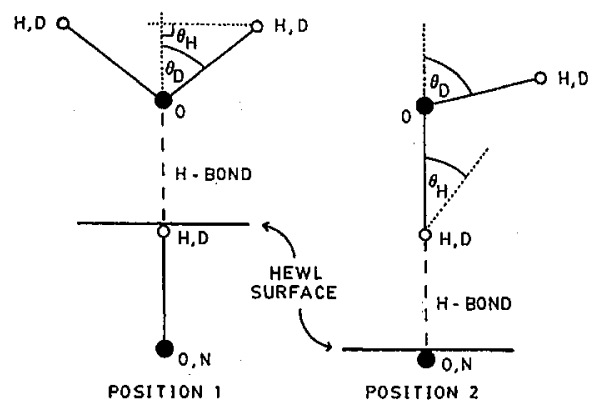

Fig. 10. Hydrogen bond configurations of water molecules on the HEWL surface. For position 1: It is assumed that in this case the effective position of the water molecule is such that the hydrogen bond and the water molecule's twofold axis of symmetry are colinear; thus $\theta_{\mathrm{H}}=90^{\circ}, \theta_{\mathrm{D}}=52.5^{\circ}$. For position $2: \theta_{\mathrm{H}}=37.5^{\circ}, \theta_{\mathrm{D}}=75^{\circ}, 0^{\circ}$.

To calculate the ${ }^{1} \mathrm{H}$ rates, using the information from the ${ }^{2} \mathrm{H}$ results, Eq. (5) was fitted first to the ${ }^{2} \mathrm{H}$ data (Fig. 6b) with $f_{\mathrm{wb}}$ and $\tau_{f}$ as adjustable parameters and $R_{1 \mathrm{wb}}$ of Eq. (8). Then these parameters were used to calculate the ${ }^{1} \mathrm{H}$ rates using the same equations. In these calculations the appropriate angles (Fig. 10), constants, and free water rates were applied. In addition a value for $\tau_{s}=8 \times 10^{-9} \mathrm{~s}$ for the protein tumbling time was assumed (Table III).

The procedure was repeated for different values of $f_{1}$ until a reasonable correspondence was obtained between the calculated intrinsic proton rates at $5 \mathrm{MHz}$ and at $30.6 \mathrm{MHz}$ and those found in the isotopic dilution study (Fig. 9). The calculated rate at $30.6 \mathrm{MHz}$ was found to be relatively insensitive to changes in $f_{1}$. Therefore, $f_{1}$ was finally adjusted such that the calculated value at $5 \mathrm{MHz}$ matched with $R_{1 \mathrm{w}}$ from the isotopic dilution study at the same frequency. The final calculated values are shown in Fig. 9 (dashed line) with adjustable parameters given in the figure caption. It should be noted that the fit to the deuteron data (dashed-dotted line, Fig. 9), from which the adjustable parameters were obtained, is excellent.

The dispersive behaviour of the contribution of the water proton - HEWL proton intermolecular coupling to the observed rate of the $L_{1}$-component in $\mathrm{N}$-HEWL is obtained by taking the differences between the $L_{1}$-component rates and the intrinsic water proton rates (dashed line, Fig. 9). These difference rates are shown in Fig. 11. 


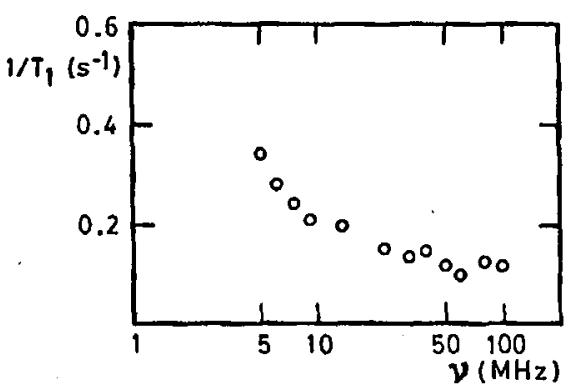

Fig. 11. The water proton - HEWL proton intermolecular contribution (difference rates) in N-HEWL.

It is extremely interesting that the intermolecular water-HEWL contribution decreases with increasing frequency (Fig. 11) in a fashion not unlike that of a Debye function (such as in Eq. (2) for example). As discussed in the next section, we interpret this contribution as being caused by a water-HEWL interfacial spin-lattice relaxation mechanism.

According to the isotropic motion model, only about 3 water molecules per HEWL molecule are required to explain the rates $R_{1 \mathrm{wb}}$. An application of the more realistic anisotropic motion model yields $f_{\mathrm{wb}}=(3.8 \pm 0.6) \times 10^{-3}$ (Fig. 9), or about 15 water molecules per HEWL molecule. These numbers of water molecules are very small compared with the number of water molecules that constitutes a monolayer (160), and would necessitate the existence of a few very special water molecule binding sites on the HEWL molecule.

Similar conclusions were reached by Koenig et al. [24-26] from proton and deuteron relaxometry of protein solutions. These authors proposed the existence of a very limited number of water molecules which are bound via multiple hydrogen bonds to protein surface sites. The very close association of a small number of water molecules (relative to a monolayer) with protein molecules has also been proposed by Denisov and Halle [27, 28] and Venu et al. [29]. These authors indicate that such water molecules are buried inside the protein and exchange with bulk water on a time scale at least as long as the protein tumbling time.

\subsection{Model for intermolecular water proton-HEWL proton coupling}

Although it has been indicated that exchange of labile protein hydrogens can make an important contribution to the solvent spin relaxation [27-29], this effect is negligible in this solution [33]. We explore the details of the intermolecular coupling between water protons and HEWL protons, and of the associated water molecule dynamics, using a cross-relaxation model [40]. This model treats the protein protons and water protons at the interface using Solomon's coupled two-spin-system approach [49] as outlined by Fung and McGaughy [41]. In this approach $R_{1 \mathrm{w}}$ and $R_{1 \mathrm{p}}$ are each weighted sums of three contributions: the intrinsic spin-lattice relaxation rates of the uncoupled water protons $\left(R_{1 \mathrm{wf}}\right)$ and protein protons $\left(R_{1 \mathrm{pf}}\right)$, the corresponding spin-lattice relaxation rates of the protons coupled at the interface 
( $R_{1 \mathrm{wb}}$ and $R_{1 \mathrm{pb}}$, respectively), and the contribution of the mutual interaction of the coupled protons to the spin-lattice relaxation rate $\left(R_{1 \mathrm{w}-\mathrm{p}}\right)$. Thus

$$
R_{1 \mathrm{i}}=\left(1-f_{\mathrm{ib}}\right) R_{1 \mathrm{if}}+f_{\mathrm{ib}}\left(R_{1 \mathrm{ib}}+R_{1 \mathrm{w}-\mathrm{p}}\right)
$$

where $\mathrm{i}=\mathrm{w}$ or $\mathrm{p}$ and $f_{\mathrm{pb}}$ is the fraction of protein protons sharing an intermolecular relaxation with water protons at the interface.

In the presence of cross-relaxation between the HEWL and water protons two-component behaviour may be observed in the magnetization recovery curves with the observed, apparent relaxation rates given $[40,41]$ by

$$
\begin{aligned}
2 \lambda^{ \pm} & =R_{1 \mathrm{w}}+R_{1 \mathrm{p}}+k\left(f_{\mathrm{pb}}+f_{\mathrm{wb}}\right) \\
& \pm\left\{\left[R_{1 \mathrm{p}}-R_{1 \mathrm{w}}+k\left(f_{\mathrm{pb}}-f_{\mathrm{wb}}\right]^{2}+4 k^{2} f_{\mathrm{pb}} f_{\mathrm{wb}}\right\}^{1 / 2},\right.
\end{aligned}
$$

where the cross-relaxation rate $k$ is given [49] by

$$
k=\frac{\gamma^{4} \hbar^{2}}{10 r_{i j}^{6}}\left[\tau_{\mathrm{c}}-\frac{6 \tau_{c}}{1+4 \omega^{2} \tau_{c}^{2}}\right]
$$

with $r$ the water proton - protein proton internuclear distance and $\tau_{\mathrm{c}}$ the correlation time characterizing the reorientation of the associated vector. According to this model, $R_{1 \mathrm{w}-\mathrm{p}}$ and $k$ are related through the transition probabilities (W) of a two-spin system [49].

In N-HEWL we associate $\lambda^{-}$, the inverse time constant of the slow relaxing magnetization component, with the $L_{1}$-component. The applicability of a cross-relaxation model to N-HEWL can then be explored by determining if values of $\lambda^{-}$calculated from Eq. (10) can be used to predict the experimental rates of the $L_{1}$-component in N-HEWL.

For the purpose of the following calculation we assume that $R_{1 \mathrm{pb}}=R_{1 \mathrm{pf}}$. Then $R_{1 \mathrm{w}}$ and $R_{1 \mathrm{p}}$ of Eqs. (9) can be replaced with

$$
R_{1 \mathrm{w}}=R_{\mathrm{WATER}}+f_{\mathrm{wb}} R_{1 \mathrm{w}-\mathrm{p}}
$$

and

$$
R_{1 \mathrm{p}}=R_{\text {PROTEIN }}+f_{\mathrm{pb}} R_{1 \mathrm{w}-\mathrm{p}},
$$

where the values of $R_{\text {WATER }}$ are taken from Fig. 9 (dashed line - calculated from

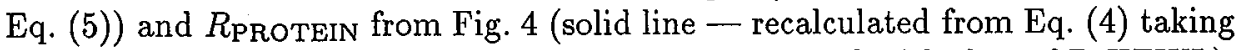
into account the smaller viscosity of N-HEWL compared with that of D-HEWL).

It is assumed that a particular proton at the interface interacts with only one proton across the interface at a distance of $2.5 \AA$ (a typical hydrogen bond length [50]). In addition, we let the water molecules involved in water-HEWL intermolecular communication be irrotationally bound, so that they undergo isotropic motion as the protein tumbles in solution. The characteristic time for this motion is taken as $8 \mathrm{~ns}$, a typical protein tumbling time (see Table III). The use of this isotropic motion model will have the result of predicting intermolecular relaxation effects (across the interface) that are somewhat larger than would be obtained upon application of the anisotropic motion model. The use of the protein tumbling time is expected to represent an upper limit $\tau_{\mathrm{c}}$ characterizing the motion of the HEWL proton - water proton vector. 
The values of $\lambda^{-}$were calculated from Eq. (10) considering that about 15 water molecules per HEWL molecule are involved in spin-flip communication across the interface as suggested by the above calculations involving the anisotropic motion model. Such values of $\lambda^{-}$were found to differ little from $R_{\text {WATER }}$ in the frequency range of present experiments.

It is apparent from Eq. (10) (with $R_{1 \mathrm{w}}$ from Eq. (11)) and the foregoing discussion that in order to substantially increase the effects of $R_{1 \mathrm{w}-\mathrm{p}}$ or $k$ it is necessary for a much larger number of water molecules than that corresponding to $f_{\mathrm{wb}}$ to be involved in the corresponding interactions. The maximum number of such water molecules would not be expected to exceed that associated with a monolayer coverage $\left(0.3 \mathrm{~g} \mathrm{H}_{2} \mathrm{O} / \mathrm{g} \mathrm{HEWL}\right)$. Proceeding as above we calculated $\lambda^{-}$ using a bound water fraction associated with a monolayer coverage, letting $r$ and $\tau_{c}$ be adjustable parameters (solid line, Fig. 9). The interproton distance across the interface of $2.5 \AA$ used in this calculation is physically reasonable in view of the fact that a typical hydrogen bond length is $2.5 \AA$. In addition, the effective correlation time of $(1.6 \pm 0.3) \mathrm{ns}$ (Fig. 9) characterizing the motion of the water molecule - HEWL molecule interproton vector is somewhat less than the protein tumbling time (Table III). This is consistent with the idea that the water molecules in question are relatively mobile while at protein hydration sites.

In order to simultaneously satisfy the deuteron and proton dispersion results ( $\tau$ values and magnitudes of relaxation rates) a relatively small number of water molecules must be intimately associated with the HEWL molecule for a time at least as long as the protein tumbling time. The existence of such water molecules has been reported [24-29] in the literature. In addition, the dynamics of a relatively large number (about that of a monolayer) of bound water molecules must be such that (a) a mechanism giving rise to $R_{1 \mathrm{w}-\mathrm{p}}$ (and/or $k$ ) is possible for the proton case and that (b) this bound water does not contribute significantly to the water deuteron rate.

Requirements (a) and (b) are satisfied if the water molecules in question undergo rapid isotropic rotational diffusion (not very different from that in the bulk liquid), but are restricted in their translational motion on the HEWL molecule surface by the surface itself and by other water molecules in the first hydration layer. As a result of the rapid rotational diffusion the water intramolecular relaxation $\left({ }^{2} \mathrm{H}\right.$ or $\left.{ }^{1} \mathrm{H}\right)$ is not directly influenced by the protein dynamics. As a result of the restricted translational diffusion the dynamics of the water proton - HEWL surface proton vector contains a component that is governed by the protein tumbling time and which gives rise to a spin-lattice relaxation mechanism that contributes to $R_{1 \mathrm{w}-\mathrm{p}}$.

\section{Conclusions}

It was found that in N-HEWL the magnitudes of the magnetization components found in the $T_{2}$ experiments and in the $T_{1}$ experiments are in excellent agreement. The sizes of fractions of the $L_{1^{-}}$and $S L$-magnetization components from the $T_{1}$ dispersion measurements were found to be independent of frequency. In addition, the water and HEWL magnetization sizes predicted from the known sample composition and known spin densities of water and HEWL compare very 
well with the sizes of the $L_{1^{-}}$and $S L$-magnetization components in N-HEWL, respectively.

The above observations demonstrate that the magnetizations of the water and HEWL protons are not strongly coupled. Nevertheless, the isotopic dilution experiment showed that a considerable contribution to the observed relaxation rates comes from the interaction between water protons and nonexchangeable protein protons. This intermolecular (water-protein) contribution varies with frequency: Its dispersion is similar to the dispersion of the spin-lattice relaxation rate of the protein protons.

The dispersion results and dilution results were correlated in a model of bound water molecule motion in which the translational and rotational degrees of freedom are not coupled. According to this model only a small number of water molecules $(\sim 15$ per protein molecule) within the first monolayer are bound to the protein at specific sites. These hydrogen bonded water molecules give rise to the dispersion of the intrinsic water proton rate in N-HEWL. The remaining water molecules making up the first hydration shell reorient rapidly. Their motion is however correlated with the motion of the protein. The dynamics of water proton - protein proton vectors contains a component which mimics the tumbling motion of the protein.

\section{Acknowledgments}

This work was supported by the Natural Sciences and Engineering Research Council of Canada and by the Research Program Division of Health and Welfare Canada.

\section{References}

[1] F. Franks, Phil. Trans. R. Soc. Lond. B 278, 89 (1977).

[2] R.S. Balaban, T.L. Cehler, Magn. Reson Q. 8, 116 (1992).

[3] O.K. Daszkiewicz, J.W. Hennel, B. Lubas, Nature 200, 1006 (1963).

[4] J.A. Glasel, Nature 220, 1124 (1968).

[5] S.H. Koenig, W.E. Schillinger, J. Biol. Chem. 244, 3283 (1969).

[6] B. Blicharska, Z. Florkowski, J.W. Hennel, G. Held, F. Noack, Biochim. Biophys. Acta 207, 381 (1970).

[7] R. Kimmich, F. Noack, Z. Naturforsch. A 25, 1680 (1970).

[8] J. Oakes, J. Chem. Soc. Farad. Trans. I. 72, 216 (1976).

[9] B.D. Hilton, R.G. Bryant, J. Magn. Reson. 24, 343 (1976).

[10] A. Kalk, H.J.C. Berendsen, J. Magn. Reson. 24, 343 (1976).

[11] L. Grösch, F. Noack, Biochim. Biophys. Acta 453, 218 (1976).

[12] S.H. Koenig, R.G. Bryant, K. Hallenga, G.S. Jacob, Biochemistry 17, 4348 (1978).

[13] B.D. Sykes, W.E. Hull, G.H. Snyder, Biophys. J. 21, 137 (1978).

[14] E. Gaggelli, E. Tiezzi, G. Valensin, Chem. Phys. Lett. 63, 155 (1979).

[15] R. Kimmich, Bull. Magn. Reson. 1, 195 (1980).

[16] G. Valensin, N. Niccolai, Chem. Phys. Lett. 79, 47 (1981). 
[17] M. Eisenstadt, Biophys. J. 33, 469 (1981).

[18] M. Rydzy, W. Skrzynski, Biochim. Biophys. Acta 705, 33 (1982).

[19] F. Winter, R. Kimmich, Biochim. Biophys. Acta 719, 292 (1982).

[20] J.C. Gore, M.S. Brown, J.M. Armitage, Magn. Reson. Med. 9, 333 (1989).

[21] C. Coolbangh Lester, R.G. Bryant, Magn. Reson. Med. 22, 143 (1991).

[22] T.L. Ceckler, S.D. Wolf, V. Yip, S.A. Simon, R.S. Balaban, J. Magn. Reson. 98, 637 (1992).

[23] R.D. Brown III, S.H. Koenig, Magn. Reson. Med. 28, 145 (1992).

[24] S.H. Koenig, R.D. Brown III, R. Ugolini, Magn. Reson. Med. 29, 77 (1993).

[25] S.H. Koenig, R.D. Brown III, Magn. Reson. Med. 30, 685 (1993).

[26] S.H. Koenig, Biophys. J. 69, 593 (1995).

[27] V.P. Denisov, B. Halle, J. Mol. Biol. 245, 682 (1995).

[28] V.P. Denisov, B. Halle, J. Mol. Biol. 245, 698 (1995).

[29] K. Venu, V.P. Denisov, B. Halle, J. Am. Chem. Soc. 119, 3122 (1997).

[30] H. Peemoeller, M.M. Pintar, J. Magn. Reson. 41, 358 (1980).

[31] H. Peemoeller, R.K. Shenoy, M.M. Pintar, J. Magn. Reson. 45, 193 (1981).

[32] P.R. Bevington, Data Reduction and Error Analysis for the Physical Sciences, McGraw-Hill Book Company, New York 1969, p. 204.

[33] S.H. Koenig, K. Hallenga, M. Shporer, Proc. Nat. Acad. Sci. 72, 266r (1975).

[34] R.E. Canfield, A.K. Liu, J. Biol. Chem. 240, 1997 (1965).

[35] A. Abragam, The Principles of Nuclear Magnetism, Oxford University Press, Oxford 1961, p. 264.

[36] M. Buck, J. Boyd, C. Redfield, D.A. MacKenzie, D.J. Jeenes, D.B. Archer, C.M. Dobson, Biochemistry 34, 4041 (1995).

[37] R.C. Hardy, R.L. Cottington, J. Chem. Phys. 17, 509 (1949).

[38] J.C. Hindman, A. Svirmickas, M. Wood, J. Chem. Phys. 59, 1517 (1973).

[39] K. Hallenga, S.H. Koenig, Biochemistry 15, 4255 (1976).

[40] P.J. Andree, J. Magn. Reson. 29, 419 (1978).

[41] B.M. Fung, T.W. McGaughy, J. Magn. Reson. 39, 413 (1980).

[42] H. Peemoeller, D.W. Kydon, A.R. Sharp, L.J. Schreiner, Can. J. Phys. 62, 1002 (1984).

[43] J.C. Gore, M.S. Brown, J. Zhong, I.M. Armitage, J. Magn. Reson 83, 246 (1989).

[44] J.G. Powles, M. Rhodes, Proc. Colloq. AMPERE 14, 757 (1967).

[45] D.E. Woessner, J. Chem. Phys. 40, 2341 (1964).

[46] W.M. Shirley, R.G. Bryant, J. Am. Chem. Soc. 104, 2910 (1982).

[47] H. Peemoeller, F.G. Yeomans, D.W. Kydon, A.R. Sharp, Biophys. J. 49, 943 (1986).

[48] D.E. Woessner, J. Chem. Phys. 37, 647 (1962).

[49] I. Solomon, Phys. Rev. 99, 559 (1955).

[50] K.D. Watenpaugh, T.N. Margulis, L.C. Sieker, L.H. Jensen, J. Mol. Biol. 122, 175 (1978). 